\title{
Orbitally selective breakdown of Fermi liquid quasiparticles in $\mathrm{Ca}_{1.8} \mathrm{Sr}_{0.2} \mathrm{RuO}_{4}$
}

\author{
D. Sutter, ${ }^{1}$ M. Kim, ${ }^{2,3}$ C. E. Matt,${ }^{1}$ M. Horio, ${ }^{1}$ R. Fittipaldi, ${ }^{4,5}$ A. Vecchione,${ }^{4,5}$ V. Granata, ${ }^{4,5}$ K. Hauser, ${ }^{1}$ Y. Sassa, ${ }^{6}$ G. Gatti, ${ }^{7}$ \\ M. Grioni, ${ }^{7}$ M. Hoesch, ${ }^{8}$ T. K. Kim, ${ }^{8}$ E. Rienks, ${ }^{9}$ N. C. Plumb, ${ }^{10}$ M. Shi, ${ }^{10}$ T. Neupert, ${ }^{1}$ A. Georges, ${ }^{2,3,11,12}$ and J. Chang ${ }^{1}$ \\ ${ }^{1}$ Physik-Institut, Universität Zürich, Winterthurerstrasse 190, CH-8057 Zürich, Switzerland \\ ${ }^{2}$ College de France, F-75231 Paris Cedex 05, France \\ ${ }^{3}$ Centre de Physique Théorique, Ecole Polytechnique, CNRS, Université Paris-Saclay, F-91128 Palaiseau, France \\ ${ }^{4}$ CNR-SPIN, I-84084 Fisciano, Salerno, Italy \\ ${ }^{5}$ Dipartimento di Fisica “E. R. Caianiello,” Università di Salerno, I-84084 Fisciano, Salerno, Italy \\ ${ }^{6}$ Department of Physics and Astronomy, Uppsala University, S-75121 Uppsala, Sweden \\ ${ }^{7}$ Institute of Physics, École Polytechnique Fedérale de Lausanne (EPFL), CH-1015 Lausanne, Switzerland \\ ${ }^{8}$ Diamond Light Source, Harwell Campus, Didcot OX11 ODE, United Kingdom \\ ${ }^{9}$ Helmholtz Zentrum Berlin, Bessy II, D-12489 Berlin, Germany \\ ${ }^{10}$ Swiss Light Source, Paul Scherrer Institut, CH-5232 Villigen PSI, Switzerland \\ ${ }^{11}$ Department of Quantum Matter Physics, University of Geneva, CH-1211 Geneva 4, Switzerland \\ ${ }^{12}$ Center for Computational Quantum Physics, Flatiron Institute, 162 th Avenue, New York, New York 10010, USA
}

(Received 18 September 2018; revised manuscript received 11 March 2019; published 29 March 2019)

\begin{abstract}
We present a comprehensive angle-resolved photoemission spectroscopy study of $\mathrm{Ca}_{1.8} \mathrm{Sr}_{0.2} \mathrm{RuO}_{4}$. Four distinct bands are revealed and along the $\mathrm{Ru}-\mathrm{O}$ bond direction their orbital characters are identified through a light polarization analysis and comparison to dynamical mean-field theory calculations. Bands assigned to $d_{x z}, d_{y z}$ orbitals display Fermi liquid behavior with fourfold quasiparticle mass renormalization. Extremely heavy fermions - associated with a predominantly $d_{x y}$ band character - are shown to display non-Fermi-liquid behavior. We thus demonstrate that $\mathrm{Ca}_{1.8} \mathrm{Sr}_{0.2} \mathrm{RuO}_{4}$ is a hybrid metal with an orbitally selective Fermi liquid quasiparticle breakdown.
\end{abstract}

DOI: 10.1103/PhysRevB.99.121115

Correlated metals are typically classified either as Fermi liquids or non-Fermi liquids depending on whether or not resistivity scales with temperature squared. There is, however, transport evidence suggesting that some materials are hybrids of these two metal classes [1]. This mixed regime is of particular interest as it provides insight into how Fermi liquids break down and the nature of non-Fermi-liquid quasiparticles. In this context, multiorbital metallic systems in conjunction with strong Hund's coupling and electron correlations are of great conceptual importance [2]. Such Hund's metals are expected to display orbital differentiated quasiparticle (QP) renormalization effects along with magnetic correlations [3]. In the strongly correlated limit, orbitally selective Mott physics (OSMP) has been explored theoretically [4-9]. The concepts of Hund's metals and OSMP have both been applied to describe band structure renormalization effects in pnictide superconductor compounds [10-15]. It remains, however, unclear whether these systems exhibit genuine heavy fermion and Mott physics. In contrast, the oxide compounds $\mathrm{LiV}_{2} \mathrm{O}_{4}$ and $\mathrm{Ca}_{1.8} \mathrm{Sr}_{0.2} \mathrm{RuO}_{4}$ are multiorbital systems where the existence of heavy fermions are clearly demonstrated from specific heat measurements [16,17]. $\mathrm{Ca}_{1.8} \mathrm{Sr}_{0.2} \mathrm{RuO}_{4}$ is furthermore in close proximity to a Mott-Hubbard metal-insulator transition [18]. Angle-resolved photoemission experiments (ARPES) on this system have been interpreted in terms of both the Hund's metal and the OSMP scenario $[19,20]$. Resistivity and specific heat indicate that the ground state is a Fermi liquid (FL). However, a thermal excitation of just $1 \mathrm{~K}$ turns the system into a non-Fermi-liquid (NFL) state [16]. Here, we present a high-resolution ARPES study, demonstrating that $\mathrm{Ca}_{1.8} \mathrm{Sr}_{0.2} \mathrm{RuO}_{4}$ is neither a standard Hunds metal nor representing OSMP. In fact, the thermally excited state constitutes an example of a hybrid metal. Along the $\mathrm{Ru}-\mathrm{O}$ bond direction, bands with $d_{x z}, d_{y z}$ orbital character display FL behavior whereas $d_{x y}$-dominated bands host NFL QPs. The breakdown of FL QPs is therefore orbitally selective. This physics might apply to other ruthenate systems such as for example $\mathrm{Sr}_{3} \mathrm{Ru}_{2} \mathrm{O}_{7}$.

Single crystals of $\mathrm{Ca}_{1.8} \mathrm{Sr}_{0.2} \mathrm{RuO}_{4}$ were grown by the fluxfeeding floating-zone technique $[21,22]$. ARPES experiments were carried out at I05, SIS, $1^{3}$ beamlines of Diamond Light Source (DLS) [23], Swiss Light Source (SLS), and BESSY, respectively. All samples were cleaved in situ under UHV conditions and measured at temperatures $T=1-30 \mathrm{~K}$. ARPES spectra were collected with different incident photon energies $h v$ and light polarizations using Scienta R4000 electron analyzers. Depending on $h v$ and $T$, the overall energy resolution was in the order of $10 \mathrm{meV}$. As $\mathrm{Ca}_{1.8} \mathrm{Sr}_{0.2} \mathrm{RuO}_{4}$ has a low-temperature $\mathrm{L}$-Pbca [24] crystal structure ( $a=$ $5.33 \AA, b=5.32 \AA$, and $c=12.41 \AA$ ), orthorhombic notation is used. The electronic structure is calculated within the density functional theory+dynamical mean-field theory (DFT+DMFT) framework using WIEN2K [25] and the TRIQS library [26-28], including a strong-coupling continuous-time Monte Carlo impurity solver [29,30]. Wannier-like $t_{2 g}$ orbitals are constructed out of Kohn-Sham bands within the energy 

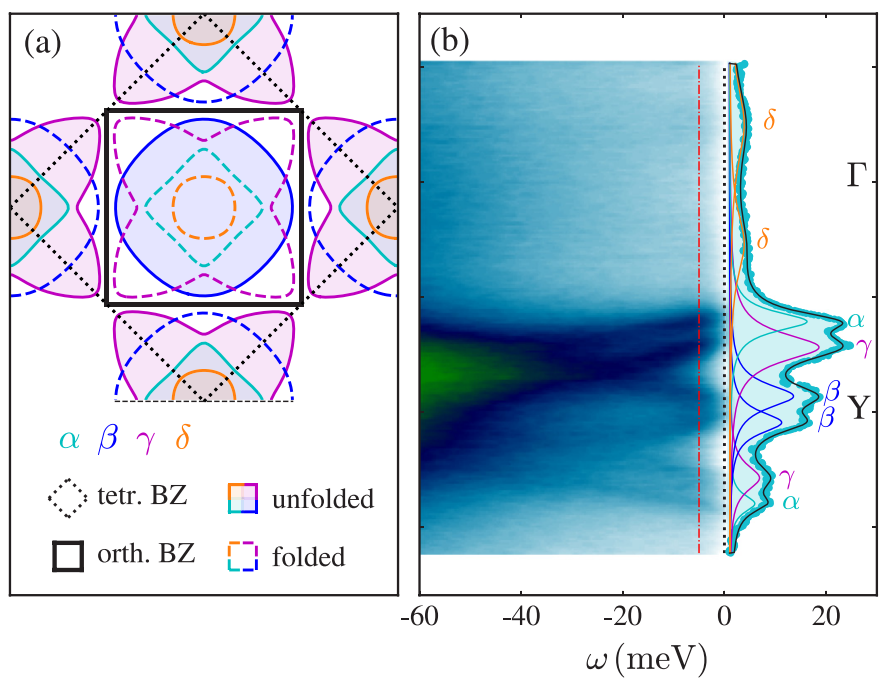
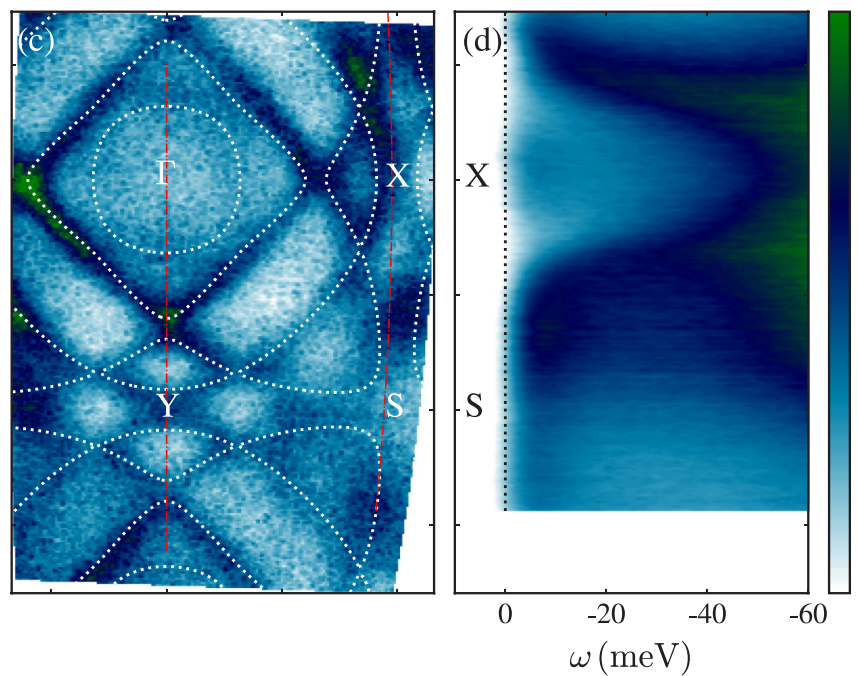

FIG. 1. Low-energy band structure of $\mathrm{Ca}_{1.8} \mathrm{Sr}_{0.2} \mathrm{RuO}_{4}$. (a) Tight-binding (TB) FS (see SM [41], which includes Refs. [31,39,42-47]) showing folded (dashed) and unfolded (solid) contours of the $\alpha, \beta, \gamma$, and $\delta$ sheets. (b) ARPES spectrum along $\Gamma-Y$ recorded with 22 -eV circularly polarized light $\left(C^{+}\right)$. Cyan circles is an MDC at fixed binding energy $5 \pm 1 \mathrm{meV}$, indicated by the red dashed line. The MDC is fitted by eight Lorentzians (total fit in black), displayed color coded to the corresponding FS sheets in (a). (c) Experimental FS map with the TB model indicated by white dots. The trajectories of ARPES spectra shown (b) and (d) are indicated by dashed red lines. (d) ARPES spectrum along the zone boundary $X-S$ showing flat bands near the Fermi level.

window $[-2,1] \mathrm{eV}$ with respect to the Fermi energy $E_{\mathrm{F}}$. For the correct description of atomic multiplets, a rotationally invariant Kanamori interaction is used [2]. Inclusion of chargeself-consistency in the DFT+DMFT loop does not change our results. This validates the correlation-induced changes of orbital occupancy in the DFT+DMFT in comparison with the DFT result.
Bulk $\mathrm{Sr}_{2} \mathrm{RuO}_{4}$ hosts three Fermi surface (FS) sheets $\alpha, \beta$ $\left(d_{x z}, d_{y z}\right)$, and $\gamma\left(d_{x y}\right)$ [31-38]. Upon Ca for Sr substitution, the $\gamma$ band is undergoing a Liftshitz transition, changing it from electron- to holelike [39]. Simultaneously, an electron pocket emerging around the zone center is predicted [40]. Orthorhombic folding of these bands [shown schematically in Fig. 1(a)] captures all the observed FS sheets of
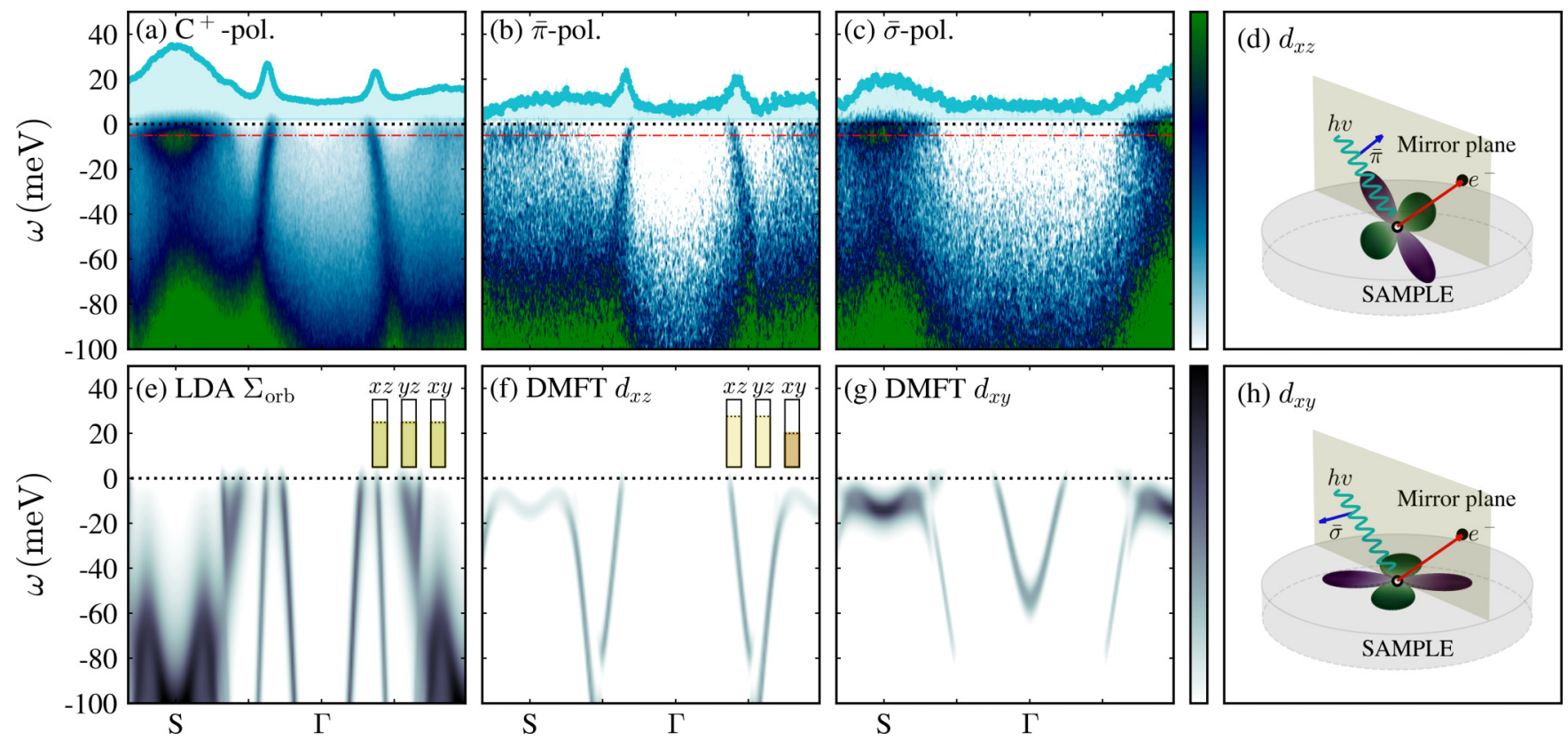

FIG. 2. Heavy fermion QPs and orbital band character. (a)-(c) ARPES spectra along the zone diagonal $(\Gamma-S)$ using $40-\mathrm{eV}$ circular, $\bar{\sigma}$, and $\bar{\pi}$ polarizations, respectively. Cyan points are MDCs near $E_{\mathrm{F}}$ (dashed turquoise lines). (d), (h) Schematics for photoemission selection rules for $d_{x y}$ and $d_{x z}$ orbitals. (e) DFT band structure along $\Gamma-S$. (f), (g) DMFT calculation of the orbitally resolved spectral function. To mimic the experimental data, the DFT and DMFT calculations are plotted in a spectral representation, truncated by the Fermi-Dirac distribution $\left(T_{\mathrm{DMFT}}=39 \mathrm{~K}\right)$, and a constant inverse lifetime of $20 \mathrm{meV}$ is used. Relative orbital fillings are indicated by the insets in (e) and (f). 


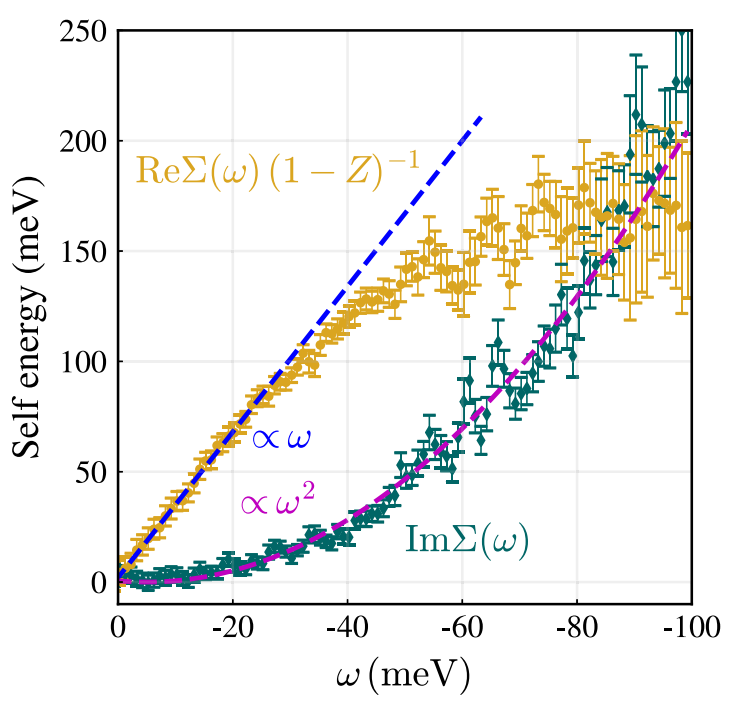

FIG. 3. Self-energy $\Sigma(\omega)$ of the $\alpha$ band, plotted as $\operatorname{Im} \Sigma(\omega)$ (green points) and $\operatorname{Re} \Sigma(\omega) /(1-Z)$ (yellow points) vs binding energy $\omega$. Dashed lines are fits to quadratic and linear dependencies, respectively. For $\operatorname{Re} \Sigma(\omega) /(1-Z)$, the fit is restricted to the low- $\omega$ regime.

$\mathrm{Ca}_{1.8} \mathrm{Sr}_{0.2} \mathrm{RuO}_{4}$. In total, four sheets are observed and labeled $\alpha, \beta, \gamma$, and $\delta$ [Figs. 1(b)-1(d)]. The weakest $\delta$ band is further documented in the Supplemental Material (SM) Fig. S1 [41] and light polarization dependence of the $\alpha$ and $\gamma$ bands is shown in Fig. 2. The $\alpha$ band, observed with $C^{+}$- and $\bar{\pi}$ polarized light, is suppressed completely in the $\bar{\sigma}$ channel. For the $\gamma$ band in the zone corner, the opposite trend is observed although complete suppression is not found. Self-energy $\Sigma(\omega)$ vs temperature and binding energy $\omega$ is extracted through a combination of momentum and energy distribution curve (EDC) analysis. For example, the $\alpha$-band QP dispersion is analyzed by fitting momentum distribution curves (MDCs). The resulting band dispersion $\varepsilon_{k}^{\alpha}$ and linewidth $\Gamma(\omega)$ led us to $\operatorname{Re} \Sigma(\omega)=\varepsilon_{k}^{\alpha}-\varepsilon_{k}^{b}$ and $\operatorname{Im} \Sigma(\omega)=\Gamma(\omega) v_{\mathrm{F}}^{b}$, where $\varepsilon_{k}^{b}$ and $v_{\mathrm{F}}^{b}$ are the DFT bare band and associated Fermi velocity (Fig. 3). The temperature dependences of the spectral intensity along the zone diagonal for both the $\alpha$ and $\gamma$ bands are analyzed in Fig. 4. In contrast to the $\alpha$ sheet, the $\gamma$-band QP peak amplitude has significant $T$ dependence.

DFT calculations provide an excellent description of the experimental FS of $\mathrm{Sr}_{2} \mathrm{RuO}_{4}$ [35]. Already without spinorbit coupling (SOC), our DFT calculation of $\mathrm{Ca}_{1.8} \mathrm{Sr}_{0.2} \mathrm{RuO}_{4}$ produces several of the experimentally observed FS sheets [Fig. 2(e)]. SOC is known to improve the calculation along the $\Gamma-Y$ direction $[35,48]$, but has no effect along the $\Gamma-S$ direction. The absence of the heavy Fermi pocket around the $S$ point in the DFT calculation is therefore a significant discrepancy [compare Figs. 2(a) and 2(e)]. This motivated our DMFT calculations, using the same parameters of the Coulomb interaction $U=2.3 \mathrm{eV}$ and Hund's coupling $J_{\mathrm{H}}=$ $0.4 \mathrm{eV}$ that successfully described $\mathrm{Ca}_{2} \mathrm{RuO}_{4}$ [49] and other ruthenates $[3,50]$. DMFT predicts strong bandwidth renormalization effects, which are particularly clear for the $\delta$ band [see Fig. 2(g)]. Moreover, our DMFT calculation reproduces qualitatively the heavy fermion states around the zone corner.

Next, we discuss the orbital character of the $\alpha$ and $\gamma$ bands along $\Gamma-S$. The incident light and center of our analyzer slit define a mirror plane to which the electromagnetic field has
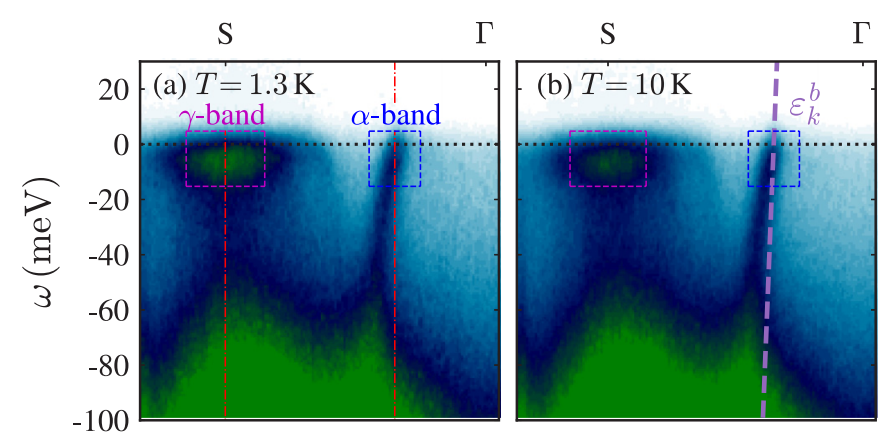

$\Gamma$
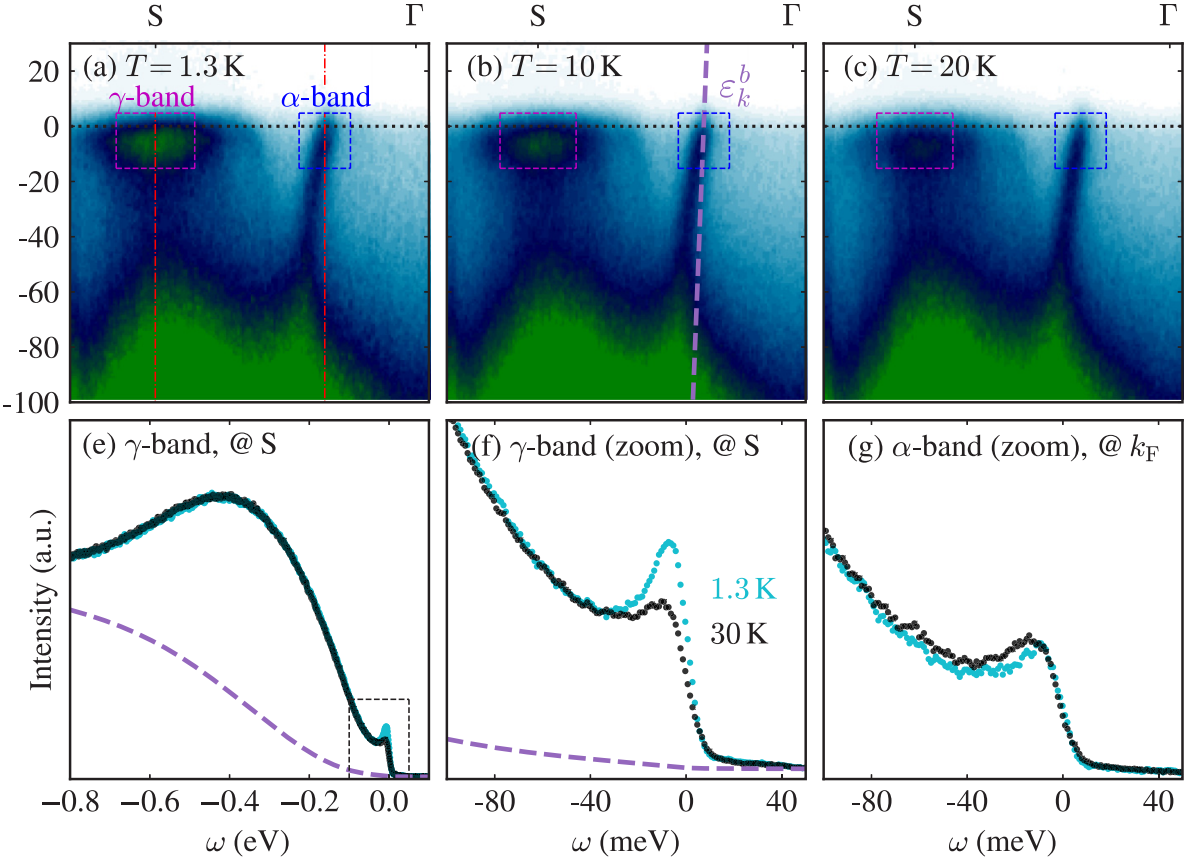

$\mathrm{S}$
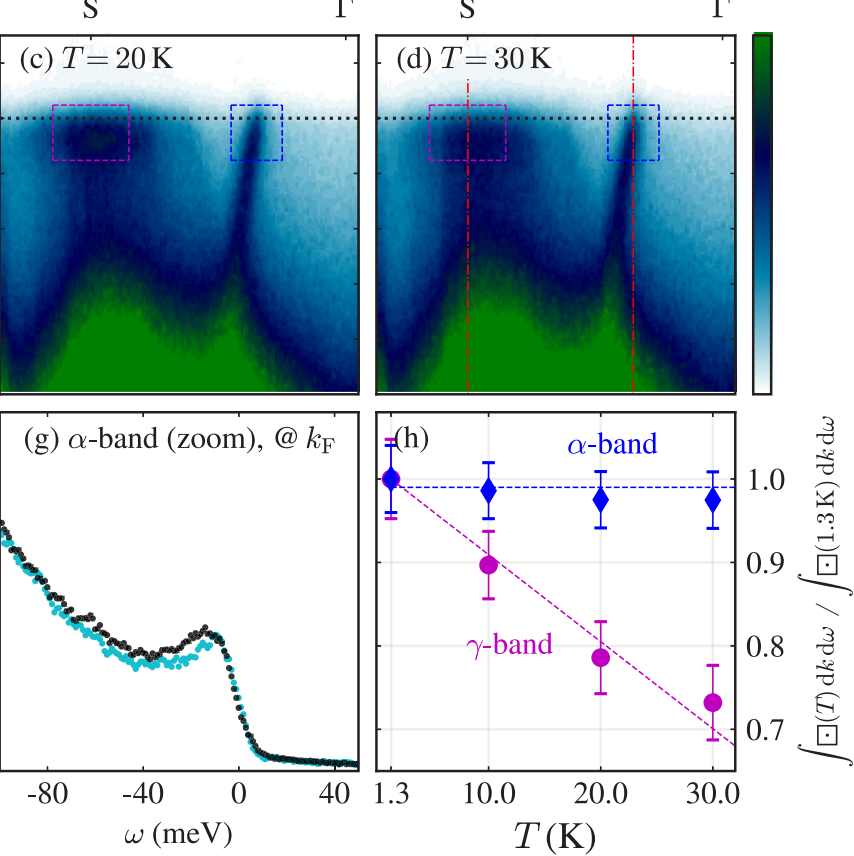

FIG. 4. Temperature dependence of QP spectral weight. (a)-(d) ARPES spectra along $S$ - $\Gamma$ for temperatures as indicated with DFT bare band dispersion $\varepsilon_{k}^{b}$ in (b). (e)-(g) Raw data EDCs of the $\alpha$ and $\gamma$ band at $T=1.3 \mathrm{~K}$ (cyan) and $30 \mathrm{~K}$ (black) and fixed momenta indicated by dashed vertical lines in (a) and (d). (f) is a zoom near $E_{\mathrm{F}}$ of the EDC displayed in (e). Dashed lines in (e) and (f) indicate a Shirley background. (h) Normalized spectral weight, integrated within the magenta $(\gamma)$ and blue $(\alpha)$ boxes shown in (a)-(d), vs $T$. 
odd (even) parity for $\bar{\sigma}(\bar{\pi})$ polarization [see Figs. 2(d) and 2(h)]. For final states with even character, selection rules [51] dictate that the odd (even) band character is suppressed in the $\bar{\pi}(\bar{\sigma})$ polarization channel. The $\alpha$ band being suppressed completely (see Fig. 2) in the $\bar{\sigma}$ channel therefore has an even character. Assuming an approximately tetragonal crystal structure, $d_{x y}, d_{x z}$, and $d_{y z}$ respectively have an odd, even, and odd character along the $a$ axis. As a result and consistent with the DFT and DMFT calculations, the $\alpha$ band along the Ru-O bond direction has pure $d_{x z}$ character. The $\gamma$ band is placed farther away from the mirror plane due to the perpendicular electron analyzer slit. Hence, less strict selection rules are expected. Nevertheless, our experimental results and DMFT calculations both assign a predominately $d_{x y}$ character to the $\gamma$ band.

As Hund's coupling quenches interorbital fluctuations, the orbitals can be viewed approximately as single bands $[2,52,53]$. For the ruthenates, this is valid only along the $\Gamma-S$ direction as the spin-orbit interaction mixes orbital characters along $\Gamma-Y$ [35]. Experimentally, it is thus only sensible to evaluate orbital differentiated properties along $\Gamma-S$. A comparison of DFT and DMFT suggests that the $d_{x y}$-dominated $\delta$ and $\gamma$ bands are most strongly affected by electron correlations. It suggests that electron correlations are orbitally differentiated. Orbital fillings provide some insight into this effect. As the $\mathrm{RuO}_{4}$ octahedron is almost cubic, DFT yields essentially degenerate $d_{x y}, d_{x z}$, and $d_{y z}$ orbital energies with equivalent 4/3 filling [inset of Fig. 2(e)]. The DMFT calculations, by contrast, indicate that electron interactions favor a less populated $d_{x y}$ orbital with $\left(n_{x y}, n_{x z}, n_{y z}\right)=(1.18,1.42,1.42)$ [inset of Fig. 2(f)]. Electron interactions thus push the $d_{x y}$ channel closer to half filling and effectively into a more correlated regime.

To describe the orbitally differentiated self-energy of $\mathrm{Ca}_{1.8} \mathrm{Sr}_{0.2} \mathrm{RuO}_{4}$, we distinguish between saturated and unsaturated FLs. The latter refers to QPs for which $Z$ has $\omega$ or temperature dependence. This implies a NFL self-energy, i.e., nonlinear $\operatorname{Re} \Sigma(\omega)$ for $\omega \rightarrow 0$. The saturated regime, by contrast, refers to a standard FL with self-energy $\Sigma(\omega, T)=$ $\gamma_{0} \omega+i \alpha_{0}\left[\omega^{2}+\left(\pi k_{\mathrm{B}} T\right)^{2}\right]$, where $\gamma_{0}$ and $\alpha_{0}$ are constants $[51,54]$. Hence, the QP residue $Z \equiv\left[1-\partial_{\omega} \operatorname{Re} \Sigma(\omega)\right]^{-1}=$ $\left(1-\gamma_{0}\right)^{-1}$ is independent of $\omega$ and $T$. A FL is therefore expected to display (1) a linear QP dispersion, (2) a linewidth that scales as $\omega^{2}, Z|\operatorname{Im} \Sigma(\omega)|<\omega$ [55] below a cutoff energy scale. and (4) a QP amplitude proportional to $Z$, independent of $\omega$ and $T$. Using Re $\Sigma(\omega)=\omega(1-1 / Z)$, the third criterion can be rewritten as $|\operatorname{Im} \Sigma(\omega)|<\operatorname{Re} \Sigma(\omega) /(1-Z)$. For criterion No. (4), the Fermi-Dirac distribution combined with finite instrumental resolution may induce a weak temperature dependence on the effectively observed QP peak amplitude. This weak effect is discussed in the SM Note I [41]. Examination of the $\alpha$ band, with pure $d_{x z}, d_{y z}$ character, reveals an almost $T$-independent QP amplitude [Fig. 4(g)]. The QP dispersion is approximately linear $\varepsilon_{k}^{\alpha} \approx v_{\mathrm{F}}\left|k-k_{\mathrm{F}}\right|$, implying $\operatorname{Re} \Sigma(\omega)=$ $\left(1-v_{\mathrm{F}}^{b} / v_{\mathrm{F}}\right) \omega$, with $v_{\mathrm{F}}$ and $v_{\mathrm{F}}^{b}$ being dressed and bare Fermi velocities [56]. Assuming an isotropic FL and using $v_{\mathrm{F}}^{b}=$ $2.34 \mathrm{eV} \AA$, the $\mathrm{QP}$ residue yields $Z=v_{\mathrm{F}} / v_{\mathrm{F}}^{b}=0.26(4)$, consistent with DMFT that finds $Z_{x z}=0.23$. Analysis of the MDC linewidth [half width at half maximum (HWHM)] at
$T=30 \mathrm{~K}$ yields $\Gamma(\omega)=\Gamma_{0}+\eta \omega^{2}$, with $\Gamma_{0}=0.020(2) \AA^{-1}$ and $\eta=10.6(6) \AA^{-1} \mathrm{eV}^{-2}$ being constants. This is documented by plotting $\operatorname{Im} \Sigma(\omega)=\left(\Gamma(\omega)-\Gamma_{0}\right) v_{\mathrm{F}}^{b}$ vs $\omega$ (Fig. 3). By comparing $\operatorname{Re} \Sigma(\omega) /(1-Z)$ and $\operatorname{Im} \Sigma(\omega)$, criterion No. (3) is obviously satisfied, and as shown in Figs. 4(g) and 4(h) the quasiparticle amplitude is temperature independent. The QP excitations of the $\alpha$ band thus fulfill, in the most strict sense, all criteria of a FL (see also SM Fig. S5 [41]).

Resistivity and specific heat measurements, however, display FL behavior for $T<1 \mathrm{~K}$ only and much heavier QP masses [16]. Reconciliation is reached by analysis of the extremely dressed $\gamma$-band QP states around the $S$ point. These QP amplitudes are roughly proportional to $Z$. In contrast to the $\alpha$ band, the QP peak amplitude of the $\gamma$ band exhibits a pronounced suppression with increased $T$ [Figs. 4(e)-4(g)]. To circumvent the effects of (i) the Fermi-Dirac distribution, (ii) impurity scattering, and (iii) finite instrumental resolution (see SM Figs. S3 and S4 [41]), it is useful to perform a box integration of spectral weight around $k_{\mathrm{F}}$ [see Figs. 4(a)4(d)]. Again, the $\gamma$ band displays a pronounce spectral weight temperature dependence whereas the $\alpha$ band remains approximately unchanged. As both the $\alpha$ and $\gamma$ bands are measured simultaneously, this effect is not a result of surface degradation. We are thus led to conclude that the $d_{x y}$-dominated $\gamma$-band states display nonsaturated FL behavior. Furthermore, the ratio between coherent and incoherent spectral weight [see Fig. 4(e)] indicates that $Z \ll 1$ around the $S$ point, in accordance with the DMFT value $Z_{x y} \approx 0.05$. We have thus demonstrated that the QP mass renormalization and FL QP breakdown are orbitally selective along the $\Gamma-S$ direction. It is also worth noticing that temperature-dependent spectral weight has also been reported in $\mathrm{CeCoIn}_{5}$ [57] and $\mathrm{Ce}_{2} \mathrm{PdIn}_{8}$ [58]. This effect may therefore be generic to heavy fermion quasiparticles.

In summary, we have presented a combined ARPES, DFT, and DMFT study of $\mathrm{Ca}_{1.8} \mathrm{Sr}_{0.2} \mathrm{RuO}_{4}$. Our results revealed the complete low-energy electronic structure. Through light polarization analysis and band structure calculations, insight into the orbital band character was obtained. By studying self-energy effects, it was demonstrated that QP masses and the FL breakdown are orbitally selective. $\mathrm{Ca}_{1.8} \mathrm{Sr}_{0.2} \mathrm{RuO}_{4}$ thus constitutes a unique example of a hybrid metal hosting orbitally differentiated FL and NFL QPs. As an outlook, it is interesting to consider the idea that NFL behavior found in $\mathrm{Ba}_{2} \mathrm{RuO}_{4}$ [59] and $\mathrm{Sr}_{2} \mathrm{RuO}_{4}$ [60] under strain has a similar underlying origin.

D.S., M.H., T.N., and J.C. acknowledge support by the Swiss National Science Foundation and Y.S. was supported by the Wenner-Gren foundation. Experiments were carried out on the I05, SIS, and $1^{3}$ endstations at the Diamond Light Source, Swiss Light Source and BESSY, respectively. We acknowledge Diamond Light Source for time on beamline I05 under Proposal No. SI15296. A.G. and M.K. acknowledge the support of the European Research Council Grant No. ERC319286-QMAC and the Swiss National Science Foundation (NCCR MARVEL), as well as support from the CPHT computer team. The Flatiron Institute is supported by the Simons Foundation. We thank all beamline staff for technical support. 
[1] R. A. Cooper, Y. Wang, B. Vignolle, O. J. Lipscombe, S. M. Hayden, Y. Tanabe, T. Adachi, Y. Koike, M. Nohara, H. Takagi, C. Proust, and N. E. Hussey, Science 323, 603 (2009).

[2] A. Georges, L. de' Medici, and J. Mravlje, Annu. Rev. Condens. Matter Phys 4, 137 (2013).

[3] J. Mravlje, M. Aichhorn, T. Miyake, K. Haule, G. Kotliar, and A. Georges, Phys. Rev. Lett. 106, 096401 (2011).

[4] V. I. Anisimov, I. A. Nekrasov, D. E. Kondakov, T. Rice, and M. Sigrist, Eur. Phys. J. B 25, 191 (2002).

[5] A. Koga, N. Kawakami, T. M. Rice, and M. Sigrist, Phys. Rev. Lett. 92, 216402 (2004).

[6] M. Vojta, J. Low Temp. Phys. 161, 203 (2010).

[7] L. de'Medici, A. Georges, and S. Biermann, Phys. Rev. B 72, 205124 (2005).

[8] S. Biermann, L. de' Medici, and A. Georges, Phys. Rev. Lett. 95, 206401 (2005).

[9] M. Ferrero, F. Becca, M. Fabrizio, and M. Capone, Phys. Rev. B 72, 205126 (2005).

[10] Z. P. Yin, K. Haule, and G. Kotliar, Nat. Phys. 7, 294 (2011).

[11] M. Yi, Z.-K. Liu, Y. Zhang, R. Yu, J. X. Zhu, J. J. Lee, R. G. Moore, F. T. Schmitt, W. Li, S. C. Riggs, J.-H. Chu, B. Lv, J. Hu, M. Hashimoto, S.-K. Mo, Z. Hussain, Z.Q. Mao, C.W. Chu, I.R. Fisher, Q. Si et al., Nat. Commun. 6, 7777 (2015).

[12] S. Gerber, S.-L. Yang, D. Zhu, H. Soifer, J. A. Sobota, S. Rebec, J. J. Lee, T. Jia, B. Moritz, C. Jia, A. Gauthier, Y. Li, D. Leuenberger, Y. Zhang, L. Chaix, W. Li, H. Jang, J.-S. Lee, M. Yi, G. L. Dakovski et al., Science 357, 71 (2017).

[13] P. O. Sprau, A. Kostin, A. Kreisel, A. E. Böhmer, V. Taufour, P. C. Canfield, S. Mukherjee, P. J. Hirschfeld, B. M. Andersen, and J. C. S. Davis, Science 357, 75 (2017).

[14] M. Aichhorn, S. Biermann, T. Miyake, A. Georges, and M. Imada, Phys. Rev. B 82, 064504 (2010).

[15] D.-H. Lee, Science 357, 32 (2017).

[16] S. Nakatsuji, D. Hall, L. Balicas, Z. Fisk, K. Sugahara, M. Yoshioka, and Y. Maeno, Phys. Rev. Lett. 90, 137202 (2003).

[17] S. Kondo, D. C. Johnston, C. A. Swenson, F. Borsa, A. V. Mahajan, L. L. Miller, T. Gu, A. I. Goldman, M. B. Maple, D. A. Gajewski, E. J. Freeman, N. R. Dilley, R. P. Dickey, J. Merrin, K. Kojima, G. M. Luke, Y. J. Uemura, O. Chmaissem, and J. D. Jorgensen, Phys. Rev. Lett. 78, 3729 (1997).

[18] S. Nakatsuji, V. Dobrosavljević, D. Tanasković, M. Minakata, H. Fukazawa, and Y. Maeno, Phys. Rev. Lett. 93, 146401 (2004).

[19] A. Shimoyamada, K. Ishizaka, S. Tsuda, S. Nakatsuji, Y. Maeno, and S. Shin, Phys. Rev. Lett. 102, 086401 (2009).

[20] M. Neupane, P. Richard, Z.-H. Pan, Y.-M. Xu, R. Jin, D. Mandrus, X. Dai, Z. Fang, Z. Wang, and H. Ding, Phys. Rev. Lett. 103, 097001 (2009).

[21] H. Fukazawa, S. Nakatsuji, and Y. Maeno, Physica B 281, 613 (2000).

[22] S. Nakatsuji and Y. Maeno, J. Solid State Chem. 156, 26 (2001).

[23] M. Hoesch, T. K. Kim, P. Dudin, H. Wang, S. Scott, P. Harris, S. Patel, M. Matthews, D. Hawkins, S. G. Alcock, T. Richter, J. J. Mudd, M. Basham, L. Pratt, P. Leicester, E. C. Longhi, A. Tamai, and F. Baumberger, Rev. Sci. Instrum. 88, 013106 (2017).

[24] O. Friedt, M. Braden, G. André, P. Adelmann, S. Nakatsuji, and Y. Maeno, Phys. Rev. B 63, 174432 (2001).
[25] P. Blaha, K. Schwarz, G. Madsen, D. Kvasnicka, and J. Luitz WIEN2k: An Augmented Plane Wave Plus Local Orbitals Program for Calculating Crystal Properties (Karlheinz Schwarz, TU Wien, 2001).

[26] M. Aichhorn, L. Pourovskii, V. Vildosola, M. Ferrero, O. Parcollet, T. Miyake, A. Georges, and S. Biermann, Phys. Rev. B 80, 085101 (2009).

[27] M. Aichhorn, L. Pourovskii, P. Seth, V. Vildosola, M. Zingl, O. E. Peil, X. Deng, J. Mravlje, G. J. Kraberger, C. Martins, M. Ferrero, and O. Parcollet, Comput. Phys. Commun. 204, 200 (2016).

[28] O. Parcollet, M. Ferrero, T. Ayral, H. Hafermann, I. Krivenko, L. Messio, and P. Seth, Comput. Phys. Commun. 196, 398 (2015).

[29] E. Gull, A. J. Millis, A. I. Lichtenstein, A. N. Rubtsov, M. Troyer, and P. Werner, Rev. Mod. Phys. 83, 349 (2011).

[30] P. Seth, I. Krivenko, M. Ferrero, and O. Parcollet, Comput. Phys. Commun. 200, 274 (2016).

[31] V. B. Zabolotnyy, D. V. Evtushinsky, A. A. Kordyuk, T. K. Kim, E. Carleschi, B. P. Doyle, R. Fittipaldi, M. Cuoco, A. Vecchione, and S. V. Borisenko, J. Electron Spectrosc. Relat. Phenom 191, 48 (2013).

[32] A. Damascelli, D. H. Lu, K. M. Shen, N. P. Armitage, F. Ronning, D. L. Feng, C. Kim, Z.-X. Shen, T. Kimura, Y. Tokura, Z. Q. Mao, and Y. Maeno, Phys. Rev. Lett. 85, 5194 (2000).

[33] C. Bergemann, A. P. Mackenzie, S. R. Julian, D. Forsythe, and E. Ohmichi, Adv. Phys. 52, 639 (2003).

[34] V. B. Zabolotnyy, E. Carleschi, T. K. Kim, A. A. Kordyuk, J. Trinckauf, J. Geck, D. Evtushinsky, B. P. Doyle, R. Fittipaldi, M. Cuoco, A. Vecchione, B. Büchner, and S. V. Borisenko, New J. Phys. 14, 063039 (2012).

[35] M. W. Haverkort, I. S. Elfimov, L. H. Tjeng, G. A. Sawatzky, and A. Damascelli, Phys. Rev. Lett. 101, 026406 (2008).

[36] H. Iwasawa, Y. Yoshida, I. Hase, S. Koikegami, H. Hayashi, J. Jiang, K. Shimada, H. Namatame, M. Taniguchi, and Y. Aiura, Phys. Rev. Lett. 105, 226406 (2010).

[37] H. Iwasawa, Y. Yoshida, I. Hase, K. Shimada, H. Namatame, M. Taniguchi, and Y. Aiura, Phys. Rev. Lett. 109, 066404 (2012).

[38] T. Kondo, M. Ochi, M. Nakayama, H. Taniguchi, S. Akebi, K. Kuroda, M. Arita, S. Sakai, H. Namatame, M. Taniguchi, Y. Maeno, R. Arita, and S. Shin, Phys. Rev. Lett. 117, 247001 (2016).

[39] S.-C. Wang, H.-B. Yang, A. K. P. Sekharan, S. Souma, H. Matsui, T. Sato, T. Takahashi, C. Lu, J. Zhang, R. Jin, D. Mandrus, E. W. Plummer, Z. Wang, and H. Ding, Phys. Rev. Lett. 93, 177007 (2004).

[40] E. Ko, B. J. Kim, C. Kim, and H. J. Choi, Phys. Rev. Lett. 98, 226401 (2007).

[41] See Supplemental Material at http://link.aps.org/supplemental/ 10.1103/PhysRevB.99.121115 for details concerning $\delta$-band identification, self-energy DMFT calculations, and the band structure fitting procedure. which includes Refs. [31,39,42-47].

[42] W.-C. Lee, D. P. Arovas, and C. Wu, Phys. Rev. B 81, 184403 (2010).

[43] C. M. Puetter, J. G. Rau, and H.-Y. Kee, Phys. Rev. B 81, 081105 (2010).

[44] K. K. Ng and M. Sigrist, Europhys. Lett. 49, 473 (2000).

[45] C. G. Fatuzzo, M. Dantz, S. Fatale, P. Olalde-Velasco, N. E. Shaik, B. Dalla Piazza, S. Toth, J. Pelliciari, R. Fittipaldi, A. Vecchione, N. Kikugawa, J. S. Brooks, H. M. Rønnow, 
M. Grioni, C. Rüegg, T. Schmitt, and J. Chang, Phys. Rev. B 91, 155104 (2015).

[46] T. Mizokawa, L. H. Tjeng, G. A. Sawatzky, G. Ghiringhelli, O. Tjernberg, N. B. Brookes, H. Fukazawa, S. Nakatsuji, and Y. Maeno, Phys. Rev. Lett. 87, 077202 (2001).

[47] D. P. Kingma and J. Ba, arXiv:1412.6980.

[48] M. Kim, J. Mravlje, M. Ferrero, O. Parcollet, and A. Georges, Phys. Rev. Lett. 120, 126401 (2018).

[49] D. Sutter, C. G. Fatuzzo, S. Moser, M. Kim, R. Fittipaldi, A. Vecchione, V. Granata, Y. Sassa, F. Cossalter, G. Gatti, M. Grioni, H. M. Rønnow, N. C. Plumb, C. E. Matt, M. Shi, M. Hoesch, T. K. Kim, T.-R. Chang, H.-T. Jeng, C. Jozwiak et al., Nat. Commun. 8, 15176 (2017).

[50] H. T. Dang, J. Mravlje, A. Georges, and A. J. Millis, Phys. Rev. B 91, 195149 (2015).

[51] A. Damascelli, Z. Hussain, and Z.-X. Shen, Rev. Mod. Phys. 75, 473 (2003).

[52] L. de' Medici, G. Giovannetti, and M. Capone, Phys. Rev. Lett. 112, 177001 (2014).

[53] L. de' Medici, J. Mravlje, and A. Georges, Phys. Rev. Lett. 107, 256401 (2011).

[54] C. M. Varma, Z. Nussinov, and W. van Saarloos, Phys. Rep. 361, 267 (2002).
[55] X. Deng, J. Mravlje, R. Žitko, M. Ferrero, G. Kotliar, and A. Georges, Phys. Rev. Lett. 110, 086401 (2013).

[56] C. G. Fatuzzo, Y. Sassa, M. Månsson, S. Pailhès, O. J. Lipscombe, S. M. Hayden, L. Patthey, M. Shi, M. Grioni, H. M. Rønnow, J. Mesot, O. Tjernberg, and J. Chang, Phys. Rev. B 89, 205104 (2014).

[57] Q. Y. Chen, D. F. Xu, X. H. Niu, J. Jiang, R. Peng, H. C. Xu, C. H. P. Wen, Z. F. Ding, K. Huang, L. Shu, Y. J. Zhang, H. Lee, V. N. Strocov, M. Shi, F. Bisti, T. Schmitt, Y. B. Huang, P. Dudin, X. C. Lai, S. Kirchner et al., Phys. Rev. B 96, 045107 (2017).

[58] Q. Yao, D. Kaczorowski, P. Swatek, D. Gnida, C. H. P. Wen, X. H. Niu, R. Peng, H. C. Xu, P. Dudin, S. Kirchner, Q. Y. Chen, D. W. Shen, and D. L. Feng, Phys. Rev. B 99, 081107(R) (2019).

[59] B. Burganov, C. Adamo, A. Mulder, M. Uchida, P. D. C. King, J. W. Harter, D. E. Shai, A. S. Gibbs, A. P. Mackenzie, R. Uecker, M. Bruetzam, M. R. Beasley, C. J. Fennie, D. G. Schlom, and K. M. Shen, Phys. Rev. Lett. 116, 197003 (2016).

[60] M. E. Barber, A. S. Gibbs, Y. Maeno, A. P. Mackenzie, and C. W. Hicks, Phys. Rev. Lett. 120, 076602 (2018). 